\title{
A 22-amino-acid peptide regulates tight junctions through occludin and cell apoptosis
}

\author{
Maoying Zhu ${ }^{\text {Equal first author, } 1}$, Juan Lu ${ }^{\text {Equal first author, }{ }^{1} \text {, Jianyun Shen }}{ }^{1}$, Lumin Fei $^{1}$, Deyu Chen ${ }^{\text {Corresp. } 2}$ \\ ${ }^{1}$ College of biological and food engineering, Fuyang Normal University, Fuyang, Anhui, China \\ ${ }^{2}$ College of medicine, Fuyang Normal University, Fuyang, Anhui, China \\ Corresponding Author: Deyu Chen \\ Email address: chendeyu7104@aliyun.com
}

Occludin is a structural protein of tight junctions (TJ) in the blood-testis barrier (BTB). A 22amino-acid peptide (22AA) in the second extracellular loop can reversibly regulate $\mathrm{TJ}$, but its regulatory mechanism is unknown. In this study, a 22AA-induced TJ destruction animal model was constructed to investigate the effect of 22AA on Sertoli cells (SCs) and spermatid count $\mathrm{s}$ and cell apoptosis at different time points using a multiplex immunofluorescence technique. The effect of 22AA on the location and distribution of occludin was analyzed via dual confocal fluorescence microscope. Western blotting was used to analyze dynamic changes in occludin expression. Real-time RT-PCR was used to analyze miR-122-5p expression changes. Sperm density counts and mating methods were used to analyze the effect of 22AA on fertility in mice. The results showed that 22AA promoted SC and spermatid apoptosis, downregulated occludin, upregulated miR-122-5p, and decreased sperm density and litter size before 27 days (27D). After 27D, the expression of occludin increased again, miR-122-5p expression decreased again, both sperm density and litter size returned to normal, apoptosis stopped, and spermatogenesis began to recover. Therefore, it can be concluded that 22AA can destroy TJ by downregulating occludin and inducing cell apoptosis. After 27D, TJ and spermatogenesis functions return to normal. 
1

2 Title: A 22-amino-acid peptide regulates tight junctions through occludin and cell

3 apoptosis

4 Short Title: A peptide regulates tight junctions

5 Names of each author:

1. Maoying Zhu: College of biological and food engineering,Fuyang Normal University; Fuyang Anhui 236037, China.

2. Juan Lu: College of biological and food engineering,Fuyang Normal University; Fuyang Anhui 236037,China.

3. Jianyun Shen: College of biological and food engineering,Fuyang Normal University; Fuyang Anhui 236037,China.

4. Lumin Fei: College of biological and food engineering, Fuyang Normal University; Fuyang Anhui 236037, China.

5. Deyu Chen: College of medicine, Fuyang Normal University. Fuyang Anhui 236037, China.

Corresponding author: College of medicine, Fuyang Normal University, No.100 Qinghe Road, Fuyang, Anhui 236037, China. Telephone number: 86-558-2596113. Email: chendeyu7104@aliyun.com.

19

20

21

22

\section{Title Page}




\section{A 22-amino-acid peptide regulates tight junctions through occludin and cell apoptosis}

Abstract: Occludin is a structural protein of tight junctions (TJ) in the blood-testis barrier (BTB).

A 22-amino-acid peptide (22AA) in the second extracellular loop can reversibly regulate TJ, but

its regulatory mechanism is unknown. In this study, a 22AA-induced TJ destruction animal

model was constructed to investigate the effect of 22AA on Sertoli cells (SCs) and spermatid

counts and cell apoptosis at different time points using a multiplex immunofluorescence

technique. The effect of 22AA on the location and distribution of occludin was analyzed via dual

confocal fluorescence microscope. Western blotting was used to analyze dynamic changes in

occludin expression. Real-time RT-PCR was used to analyze miR-122-5p expression changes.

Sperm density counts and mating methods were used to analyze the effect of 22AA on fertility in

mice. The results showed that 22AA promoted SC and spermatid apoptosis, downregulated

occludin, upregulated miR-122-5p, and decreased sperm density and litter size before 27 days

(27D). After 27D, the expression of occludin increased again, miR-122-5p expression decreased

41 again, both sperm density and litter size returned to normal, apoptosis stopped, and

42 spermatogenesis began to recover. Therefore, it can be concluded that 22AA can destroy TJ by

43 downregulating occludin and inducing cell apoptosis.After 27D, TJ and spermatogenesis

44 functions return to normal.

Keywords: occludin; TJ; apoptosis; Sertoli cell; spermatid 


\section{Introduction}

In the testis, there is a blood-testis barrier (BTB) between the seminiferous tubules and

blood vessels. The BTB composition includes the interstitial capillary endothelium and its

basement membrane, connective tissue, and tight junctions (TJ) between the basement

membrane of the seminiferous epithelium and Sertoli cells (SCs). TJ are the main structures that

constitute the BTB [1]. One cause of male infertility is TJ abnormality in SCs, resulting in

blockage of spermatogenic cell migration in seminiferous tubules [2]. The three-dimensional

configuration of SCs is complex. SCs are irregularly cone-shaped, with the base closely touching

the basement membrane, the top extending into the lumen, and many irregular depressions in the

lateral surface and lumen surface, in which various levels of spermatogenic cells are embedded.

Membranes near the basal side of the adjacent SCs form TJ, and the seminiferous epithelium is

divided into a basal compartment and adluminal compartment[3]. The basal compartment is

located between the basement membrane of seminiferous epithelium and the TJ of SCs and

contains spermatogonia cells (including type A and type B). The adluminal compartment, located

above the TJ, connects with the lumen of seminiferous tubules and contains spermatocytes, germ

cells, and sperm [4]. During spermatogenesis, the spermatocytes at the preleptotene stage and the

leptotene stage differentiated from type B spermatogonia cells must move across the TJ into the abluminal compartment to complete their development [5]. Therefore, the movement of germ reconstruction of TJ. 
Many TJ experimental models exist for studying the BTB. The in vitro BTB experimental model uses the testicular SC primary dual-chamber culture method to determine TJ tightness by measuring transepithelial electrical resistance [6]. Glycerol [7], $\mathrm{CdCl} 2$ [8], and blocking peptides [9] can also destroy testicular TJ. However, the destruction of testicular TJ induced by glycerin injection or $\mathrm{CdCl}_{2}$ injection is irreversible. Thus, studying the dynamic changes in TJ-related molecules during the disintegration process and reconstruction process using these two animal models is difficult.

Structural proteins of TJ include occludin [10], zonula occludens-1 (ZO-1) [11], ZO-2 [12], ZO-3, the claudin multigene family, and adhesion molecules [13]. Occludin is a $65-\mathrm{kDa}$ protein localized at TJ $[14,15]$ that consists of four transmembrane domains, a long carboxyl-terminal cytoplasmic domain, a short N-terminal cytoplasmic domain, two extracellular loops, and one intracellular loop [16]. Its structure is highly conserved among different mammalian species[17]. The first extracellular loop is rich in tyrosine and glycine, accounting for approximately $60 \%$ of the amino acid residues, and is involved in intercellular adhesion [18]. The second extracellular loop is involved TJ [19]. Occludin has also been reported to induce apoptosis and apoptotic sensitization, which are regarded as antitumorigenic activities [20]. However, the mechanism underlying the specific role of occludin in cell apoptosis remains poorly understood.

Wong and Gumbiner et al. used an in vitro cell model to add an artificially synthesized 44amino-acid short peptide identical to the second extracellular loop sequence of occludin into the Xenopus kidney epithelial cell line A6, thereby reducing the tightness of TJ [21]. Chung et al. confirmed that a 22-amino-acid peptide (22AA) in the second extracellular loop of occludin 
could reversibly regulate TJ [9]. However, Chung et al. only analyzed the effect of 22AA on the morphological structure of the seminiferous tubules. What is the mechanism underlying the regulation of TJ by 22AA? Is the expression and localization of occludin affected by 22AA? How are SC and spermatid counts in the seminiferous tubules dynamically affected by 22AA? Can 22AA affect the number of offspring? The above questions should be studied in depth. To further investigate the mechanism underlying the regulation of $\mathrm{TJ}$ by $22 \mathrm{AA}$, this study analyzed the effect of 22AA on occludin expression and localization, SC and spermatid apoptosis, and mouse fertility using a TJ damage animal model.

\section{Experimental materials and methods}

\subsection{Experimental animals}

Specific-pathogen-free Kunming (KM) mice, 6 to 8 weeks old, with a body weight of 30-35

g, were provided by Chongqing Enswell Biotechnology Co., Ltd., China. Animals were kept at a temperature of $23-25^{\circ} \mathrm{C}$ with a $12 \mathrm{~h}$ light: $12 \mathrm{~h}$ dark $(12 \mathrm{~h}-12 \mathrm{~h})$ cycle. The animals were allowed food and water ad libitum. The experiment was approved by the Experimental Animal Ethics Committee of Fuyang Normal University, China (Grant No. 20200006).

\subsection{Construction of animal models}

The sequence of 22AA is NH2-GSQIYTICSQFYTPGGTGLYVD-COOH (from the 209th to 230th amino acid of occludin) [9], and 22AA was synthesized by Beijing Protein Innovation Co., Ltd., China. To prepare a $200.0 \mathrm{~g} / \mathrm{L}$ solution, $22 \mathrm{AA}$ was dissolved in $0.9 \%$ sterile saline. Male KM mice were anesthetized with $7 \%$ chloral hydrate $(0.5 \mathrm{~mL} / 100 \mathrm{~g})$, the scrotal skin was cut, both (left and right) testicles were exposed, and a 26-gauge needle (Becton Dickinson, 
110

111

112

113

114

115

116

117

118

119

120

121

122

123

124

125

126

127

128

129

130

Rutherford, NJ) was used for injection of $7.0 \mu \mathrm{L}$ of $22 \mathrm{AA}$ solution at three different locations in each testicle. The control group was injected with $0.9 \%$ sterile saline. After injection, the testicles were sutured, and the mice were put back into the cage. Thirty-six experimental animals were divided into six groups, with six mice in each group: A, normal control group; B, 7 days (7D) after injection of 22AA; C, 17D after 22AA injection; D, 27D after 22AA injection; E, 37D after 22AA injection; and F, 47D after 22AA injection. After the mice were anesthetized by intraperitoneal injection of $7 \%$ chloral hydrate $(0.5 \mathrm{~mL} / 100 \mathrm{~g})$ at different time points, the skin of the scrotum was removed, and one testis was removed and stored at $-80^{\circ} \mathrm{C}$ for western blotting (WB) and quantitative PCR (qPCR). The other testis was fixed with 4\% paraformaldehyde for morphological examination. At the end of the experiment, the mice were euthanized using 0.3 $\mathrm{mL} \quad 7 \%$ chloral hydrate $(0.5 \mathrm{~mL} / 100 \quad \mathrm{~g})$ according to the American Veterinary Medical Association (AVMA)

Guidelines for the Euthanasia of Animals, 2013 Edition.

\subsection{Multiplex immunofluorescence detection}

Testicular tissues were collected at different stages, fixed and blocked using conventional methods, embedded in paraffin, deparaffinized, dehydrated, and subjected to heat-induced antigen retrieval. The types and sources of antibodies used in the experiments are shown in Table 1. The sections were washed with distilled water, immersed in phosphate-buffered saline (PBS) for $5 \mathrm{~min}$, and then blocked with goat serum at room temperature for $60 \mathrm{~min}$. The blocking solutions were then aspirated, primary anti-Bax antibody $(1: 200)$ was added dropwise, and the sections were placed in a humidified box and incubated at $4^{\circ} \mathrm{C}$ overnight. The next day, the 
131

132

133

134

135

136

137

138

139

140

141

142

143

144

145

146

147

148

149

150

151

sections were incubated at room temperature for $30 \mathrm{~min}$. The sections were immersed and washed in PBS three times for 3 min each. After the sections were blotted, a fluorescent Cy3labeled secondary antibody (1:800 dilution) was added dropwise, followed by incubation at $37^{\circ} \mathrm{C}$ for $60 \mathrm{~min}$ in a humidified box. The sections were washed three times with PBS for 3 min each. After the sections were blotted, primary anti-WT1 or anti-Prm2 antibody (1:100 dilution) was added dropwise, and the sections were incubated in a humidified box at $37^{\circ} \mathrm{C}$ for $60 \mathrm{~min}$. The sections were washed three times with PBS for 3 min each. After the sections were blotted, a fluorescent FITC-labeled secondary antibody (1:800) was added dropwise, followed by incubation at $37^{\circ} \mathrm{C}$ for $60 \mathrm{~min}$ in a humidified box. After three washes with PBS for 3 min each, DAPI was added dropwise, followed by incubation in the dark for $15 \mathrm{~min}$ for nuclear staining. Then, the sections were washed for 5 min four times. After excess DAPI was washed away, the slides were mounted in anti-fluorescence quencher mounting medium. The sections were then observed under a confocal fluorescence microscope (Leica, Germany), and images were collected. Fifty sections in different parts of each testicle were examined. Images were quantitatively analyzed using ImageJ software. The relative expression value of Bax was determined by the optical density value.

\subsection{Immunofluorescence analysis of occludin localization and distribution}

Testicular tissues were collected at different stages, fixed and blocked by conventional methods, embedded in paraffin, deparaffinized, dehydrated, and subjected to heat-induced antigen retrieval. The sections were cooled at room temperature for 10-20 min, rinsed with distilled water, and immersed in PBS for 5 min. Sections received 0.5\% Triton X-100 dropwise, 
152 followed by incubation at room temperature for $60 \mathrm{~min}$ and three PBS washes of $3 \mathrm{~min}$ each. The

153 sections were blocked at room temperature for $60 \mathrm{~min}$ via dropwise addition of normal goat

154 serum. The blocking solution was aspirated, and primary anti-occludin antibody (Abcam,

155 America) diluted 1:100 was added dropwise. The sections were placed in a humidified box and

156 incubated at $4^{\circ} \mathrm{C}$ overnight. The humidified box was removed and rewarmed to room

157 temperature for $30 \mathrm{~min}$. Sections were washed with PBS three times for 3 min each. After the

158 excess liquid was removed from the sections, Alexa Fluor Cy3-labeled antibody (Abcam,

159 America) diluted 1:800 was added dropwise, followed by incubation at $37^{\circ} \mathrm{C}$ for $60 \mathrm{~min}$ in a

160 humidified box. Sections were washed with PBS three times for $3 \mathrm{~min}$ each, and DAPI

161 (Beyotime, China) was added dropwise for nuclear staining in the dark for 15 min. After excess

162 DAPI was washed away, the slides were mounted using anti-fluorescence quencher mounting

163 medium. The sections were then observed under a confocal fluorescence microscope (Leica,

164 Germany), and images were collected. Fifty sections in different parts of each testicle were

165 examined.

166

167

168

169

170

171

172

\subsection{Detection of occludin via WB}

After extraction of total proteins from testes at different stages, the total protein content was determined using a BCA kit (Solarbio, China). After sodium dodecyl sulfate-polyacrylamide gel electrophoresis (SDS-PAGE) at $100 \mathrm{~V}$ for $1.5 \mathrm{~h}$, samples were transferred to a nitrocellulose (NC) membrane, which was blocked for $2 \mathrm{~h}$ via gentle shaking in $50.0 \mathrm{~mL}$ of TTBS (20.0 mmol/L Tris, pH 7.5, 0. $5 \mathrm{~g} / \mathrm{L}$ Tween-220, $8.0 \mathrm{~g} / \mathrm{L} \mathrm{NaCl}$ ) containing $50 \mathrm{~g} / \mathrm{L}$ skim milk powder. The NC membrane was placed in a plastic mantle and sealed after addition of $5.0 \mathrm{~mL}$ of rabbit 
173 anti-mouse occludin monoclonal antibody (Abcam, America) at 1:500 dilution and then

174 incubated at $4^{\circ} \mathrm{C}$ overnight. The NC membrane was taken out and washed with TTBS three

175 times for $15 \mathrm{~min}$ each. Then, $5.0 \mathrm{~mL}$ of horseradish peroxidase-conjugated goat anti-rabbit IgGI

176 (1:2 000 dilution with TBS) (Jackson, 111-035-008) was added and incubated with the

177 membrane with gentle shaking for $2 \mathrm{~h}$. The NC membrane was taken out and washed with TTBS

178 three times for $10 \mathrm{~min}$ each. novaECL reagent was added to the front surface of the NC

179 membrane and allowed to stand for $1 \mathrm{~min}$, and then, light-sensitive films were observed in a dark

180 room. The band density was scanned using a digital gel image analysis system, and the gray

181 value for density was measured with Image Lab 4.1. Using $\beta$-actin as the internal control, the

expression of occludin was determined by the ratio of the gray values of occludin to those of $\beta$ -

183 actin.

184

185

186

187

188

189

190

191

192

193

\subsection{Real-time RT-PCR analysis of miR-122-5p}

Testicular tissues were collected at different time points, and RNA was extracted using a

Trizol Total RNA Extraction Kit (Shanghai Sangong, China, catalog number: B511321).

According to the kit manual, RNA was extracted and reverse-transcribed into cDNA. The primers for miR-122-5p RT-PCR were F: CCTGGAGTGTGACAATG and R: GAGCAGGCTGGAGAA. The primers for the internal control actin were F: GAGACCTTCAACACCCCAGC and R: ATGTCACGCACGATTTCCC. The BR Green I protocol of the SYBR Green I method was used. The real-time fluorescence PCR kit TransStart Green qPCR SuperMix (catalog no. AQ131-01) was used for PCR amplification on a LightCycler 96 system (Roche, US). The reaction system was as follows: $25.0 \mu \mathrm{L}$ of $2 \times$ PCR 
194 buffer, $5.0 \mu \mathrm{L}$ of primers $(25.0 \mathrm{pmol} / \mu \mathrm{L}), 0.5 \mu \mathrm{L}$ of SYBR green I $(20 \times), 2.0 \mu \mathrm{L}$ of template 195 (cDNA), and $21.5 \mu \mathrm{L}$ of DEPC water. The amplification conditions were as follows: $94^{\circ} \mathrm{C}$ for 4 $196 \mathrm{~min} ; 35$ cycles of $94^{\circ} \mathrm{C}$ for $20 \mathrm{sec}, 60^{\circ} \mathrm{C}$ for $30 \mathrm{sec}$, and $72^{\circ} \mathrm{C}$ for $30 \mathrm{sec}$; followed by $72^{\circ} \mathrm{C}$ for 197 detection. Relative expression was calculated using the $2^{-\Delta \Delta \mathrm{Ct}}$ method.

198

199

200

201

202

203

204

205

206

207

208

209

210

211

212

213

214

\subsection{Analysis of sperm density}

Male mice were sacrificed via cervical dislocation at various time points. After abdominal disinfection, the abdominal wall was cut open to expose the reproductive system. The epididymis was separated via aseptic surgery, and the mesentery and fat surrounding the epididymis were removed with ophthalmic scissors and rinsed. The epididymis was shred and placed in a Petri dish containing $37^{\circ} \mathrm{C}$ PBS and then incubated at $37^{\circ} \mathrm{C}$ with $5 \% \mathrm{CO}_{2}$ and saturated humidity for 30 min. The sperm density was calculated using a cell counting plate after the sperm had spontaneously spread out.

\subsection{Analysis of litter size}

Male mice were co-caged with female mice (female:male $=1: 1$ ) at each time point. The time when a vaginal plug was detected was taken as the 0 day of pregnancy. On the 14 th day of pregnancy, the mice were sacrificed by cervical dislocation, the uterus was removed by laparotomy, and the number of embryos in the bilateral uterus was recorded.

\subsection{Statistical analysis}

The experimental results are expressed as the mean \pm standard deviation. GraphPad Prism 6 was used to complete the data processing. Differences between control group and experimental group were examined using a $\mathrm{t}$ - test. Differences among the six different experimental groups 
were examined using two-way ANOVA, and correlations between groups were examined with a

216 Pearson test. The significant difference level was set as $\mathrm{P}<0.01$.

\section{Results}

\subsection{AA promotes $\mathrm{SC}$ apoptosis}

Multiplex immunocytochemistry was used to analyze the effect of 22AA on SC apoptosis.

Bax was used as the cell apoptosis marker protein to calculate the SC apoptosis rate in

seminiferous tubules. WT1 was used as a marker of SCs. The multiplex immunohistochemical

results for each group are shown in Figure 1A. Many SCs were present in the seminiferous

tubules of the control group, with clear intercellular boundaries and a small amount of red Bax

distribution, and the expression level of Bax was relatively low at $11.373 \pm 10.532$. At $7 \mathrm{D}$, the

SC count in the seminiferous tubules was not significantly different from that in the control

group, and the expression level of Bax was $16.783 \pm 10.157$, which was significantly higher than

that in the control group $(\mathrm{P}<0.01)$, suggesting that cell apoptosis started in the seminiferous

tubules. At 17D and 27D, no cells with blue nuclei were found in the seminiferous tubules, and

the intercellular boundary completely disappeared. The WT1 patch was scattered throughout the

seminiferous tubules, and no cell structure was found in the tubules. The seminiferous tubules

and $30.253 \pm 12.274$ at $17 \mathrm{D}$ and $27 \mathrm{D}$, respectively, which were significantly higher than those in

the control group $(\mathrm{P}<0.01)$, indicating that at this stage all cells in the seminiferous tubules were 
236 a few SCs began to appear in the basal layer of seminiferous tubules; and TJ reconstruction

237 started. At 47D, the number of SCs and the Bax expression level were not significantly different

238 from those in the control group. These results demonstrate that 22AA promoted SC apoptosis.

\section{$239 \quad 3.222$ AA reversibly regulates spermatid apoptosis}

240 Multiplex immunofluorescence was used to analyze the effect of 22AA on spermatids. Bax

241 was used as the apoptosis marker protein to analyze spermatid apoptosis in the seminiferous

242 tubules. Prm2 was used as the spermatid marker to determine the spermatid count. The multiplex

243 immunohistochemical results for each group are shown in Figure 1B. Nearly $55 \pm 5$ spermatids

244 were found in the seminiferous tubules of the control group, and the expression level of Bax was

$24511.245 \pm 4.868$. At $7 \mathrm{D}$, the spermatid count decreased to $15 \pm 3$, and the expression of Bax

246 increased to $19.569 \pm 6.158$, suggesting gradual spermatid apoptosis. No spermatid was found in

247 the seminiferous tubules at 17D or 27D, and the expression levels of Bax increased to $23.467 \pm$

2485.327 and $31.353 \pm 13.139$, respectively, indicating complete spermatid apoptosis. At 37D, a

249 small amount of Prm2 was distributed in the seminiferous tubules, and the expression level of

250 Bax decreased to $16.362 \pm 3.267$, indicating that apoptosis had begun to terminate. At 47D, $20 \pm$

2514 spermatids were rediscovered in the seminiferous tubules, and the expression level of Bax was

$25210.176 \pm 1.682$, which was not different from the control level. This result indicates that 22AA

253 can reversibly regulate spermatid apoptosis.

3.3 22AA affects occludin localization and distribution

255

Dual immunofluorescence was used to analyze the effect of 22AA on the localization and 
257 Figure 1C. Occludin was mainly located at TJ between the basement membrane of

258 seminiferous tubules and SCs in the control group. At 7D, a small amount of occludin was 259 distributed at TJ between the basement membrane of seminiferous tubule and SCs. However, the 260 total number of cells in seminiferous tubules was less than that in the control group. At 17D and 261 27D, no cells with blue nuclei were found in the seminiferous tubules. From day 17 to day 27, 262 the expression of occludin gradually decreased. At 37D, occludin expression began to increase 263 and was found to be distributed on the basement membrane of seminiferous tubules and SCs. At 264 47D, occludin expression and distribution and the morphological structure of seminiferous tubules were highly similar to those in the control group. These results suggest that 22AA can reversibly affect the location and distribution of occludin.

\subsection{AA downregulates occludin}

To analyze the dynamic changes in the occludin expression level after TJ disintegration and reconstruction, total testicular proteins were extracted at $0,7,17,27,37$, or 47 days for western blot analysis. Representative western blot results are shown in Figure 2A. The relative occludin expression level was calculated using the occludin/ $\beta$-actin gray density ratio. The detailed values are shown in Figure 2B. In the control group, the expression value was 0.9967 . From 7D to 27D, the occludin expression level in the 22AA group gradually decreased to 0.1621 , which was only $16.26 \%$ of that in the control group. Then, the expression level of occludin gradually increased to 0.3543 at 47D, which was approximately one-third the normal expression level (35.54\%). Occludin expression was significantly different among the six groups $(\mathrm{P}<0.05)$. The results showed that the expression level of occludin in the 22AA group at each time was significantly 
278 different from that in the control group $(\mathrm{P}<0.01)$. These results indicate that 22AA can

279 reversibly regulate occludin expression.

280

281

282

283

284

285

286

287

288

289

290

291

292

293

294

295

296

297

298

\subsection{AA upregulates miR-122-5p expression, and miR-122-5p expression is negatively}

correlated with occludin expression before 27D

To analyze the dynamic expression of miR-122-5p during $\mathrm{TJ}$ disintegration and reconstruction, total RNA in testes was extracted at $0,7,17,27,37$, or 47 days. RT-PCR analysis was performed after the total RNA was reverse-transcribed into cDNA. The expression levels of miR-122-5p in each group are shown in Figure 3A. The miR-122-5p expression level in the control group was 0.0408 and increased to 0.0539 at 7D. The miR-122-5p expression level in the

22AA group at 27D was the highest at 0.1293. Then, miR-122-5p expression gradually decreased to 0.0867 at 47D but was still higher than that in the control group. The results showed that the miR-122-5p expression levels were significantly different among the six groups $(\mathrm{P}<$ 0.01). The correlation between miR-122-5p and occludin expression in each group was analyzed using Pearson correlation coefficient. The linear relationship is shown in Figure 3B. The results indicated that miR-122-5p and occludin expression are significantly negatively correlated $\left(\mathrm{R}^{2}=\right.$ -

$0.4905, \mathrm{P}<0.01)$

\subsection{AA changes the sperm count}

To analyze the effect of 22AA on sperm count, the epididymis was extracted at $0,7,17,27$, 37, or 47 days. The sperm density was analyzed after the epididymis was shredded. The results are shown in Figure 4A. The highest sperm density in the control group was $750.144 \times 10^{4} / \mathrm{mL}$, after which it gradually decreased. The sperm density decreased to $164.278 \times 10^{4} / \mathrm{mL}$ at $27 \mathrm{D}$ 
299 and then gradually increased to $283.114 \times 10^{4} \mathrm{~mL}$ at 47D. The results showed a significant

300 difference in sperm density among the six groups $(\mathrm{P}<0.01)$.

301

302

303

304

305

306

307

308

309

310

311

312

313

314

315

316

317

318

319

\subsection{AA affects litter size}

To analyze the effect of 22AA on the litter size of males, male mice were co-caged with female mice at $0,7,17,27,37$, or 47 days. The number of embryos in the uterus on the 14 th day of pregnancy was taken as a measure of fertility. The results are shown in Figure 4B. The largest litter size in the control group was 12, and then, litter size decreased gradually to 3.667 at 27D. The litter size gradually increased to seven at 47D. The results showed that the difference in litter size among the six groups was significant $(\mathrm{P}<0.01)$

\section{Discussion}

As a component of TJ, occludin is the structural basis for TJ formation between SCs in the seminiferous epithelium. The programmed opening/resealing of $\mathrm{TJ}$ ensures normal progression of spermatogenesis, and abnormal opening/resealing can affect the normal spermatogenesis process [22]. Interference with the functional status of occludin protein in testicular SCs can result in infertility. TJ of the BTB are different from TJ of the blood-brain barrier and other barriers, and the specific function of BTB TJ between SCs is related to spermatogonia cell activity and differentiation. The disintegration and reconstruction of $\mathrm{TJ}$ between $\mathrm{SCs}$ is an important process [23].

SCs are the structural basis of TJ in the testis [24]. The main functions of SCs include providing structural support, creating the BTB, participating in germ cell movement and ejaculation, and nurturing germ cells through the secretion process [25-28]. In the present study, 
320

321

322

323

324

325

326

327

328

329

330

331

332

333

334

335

336

337

338

339

340

to analyze the dynamic changes in SCs during the process of TJ disintegration and reconstruction, a 22AA-induced TJ destruction animal model was utilized, and an immuno-double-labeling technique was used for analysis. WTI was employed as an SC marker [29]. The results showed that the number of cells and the seminiferous tubule wall thickness were decreased in SCs at 7D compared with the control group. These results were consistent with those reported by Chung et al. [9]. No blue nuclei were found in the seminiferous tubules at $17 \mathrm{D}$ or $27 \mathrm{D}$, and the intercellular boundary had completely disappeared. WT1 was distributed throughout the seminiferous tubules. At 37D, a few SCs began to be found in the basal layer of the seminiferous tubules, and spermatogenesis began to recover. At 47D, there was almost no difference in SCs between the 22AA group and the control group, indicating that spermatogenesis had returned to normal. These phenomena reveal for the first time the changing pattern of SCs in the process of TJ disintegration and reconstruction. Next, dynamic changes in spermatids were analyzed using Prm2 as the spermatid marker [30] [31]. The results of multiplex immunohistochemistry showed that there were $55 \pm 5$ spermatids in the seminiferous tubules of the control group. At $7 \mathrm{D}$, the spermatid count decreased to $15 \pm 3$, suggesting that the spermatids gradually became apoptotic. No spermatids were found in seminiferous tubules at 17D or 27D. This finding indicates that the spermatids were completely apoptotic. A small amount of Prm2 was distributed in the seminiferous tubules at $37 \mathrm{D}$, suggesting that spermatogenesis had begun to recover. At $47 \mathrm{D}$, recovery of spermatids in seminiferous tubules was visible, and the spermatids numbered $20 \pm 4$, which was consistent with the experimental results of Chung et al. [9]. This finding indicates that spermatogenesis had returned to normal. These data suggest that $22 \mathrm{AA}$ mainly reduced the 
341 number of spermatids in seminiferous tubules before 27D. After 27D, the normal structure of the

342 TJ was restored, and spermatogenesis resumed.

343 The TJ-related structural proteins include ZO-1, ZO-2, and multiple claudin genes. Wong et al.

344 found that the 44-amino-acid peptide in the second extracellular loop of occludin had no effect

345 on the expression level of ZO-1, ZO-2, or cingulin in the Xenopus kidney epithelial cell line A6

346 [21]. Therefore, in the present study, only the dynamic expression of occludin was quantitatively

347 analyzed when investigating the mechanism underlying the destruction and recovery of TJ

348 induced by 22AA. The expression level of occludin in the 22AA group gradually decreased from

$3497 \mathrm{D}$ to $27 \mathrm{D}$ until reaching only $14 \%$ of that in the control group. Then, the expression level of

350 occludin gradually increased. At 47D, occludin expression recovered to approximately one-third

351 the normal expression. These results suggest that 22AA can reduce the expression level of

352 occludin at the disintegration stage of TJ. Therefore, it can be concluded that 22AA can

353 downregulate occludin, leading to disintegration of TJ. The 22AA -induced disruption in the TJ

354 barrier is possibly mediated by one of the following mechanisms. First, it might be possible that

355 SCs were using these peptides as building blocks for TJ assembly. However, because they did

356 not have the structural confirmation of the entire molecule to reinforce the TJ functionality, TJs

357 became perturbed and disrupted. Second, homotypic interactions of the synthetic peptides with

358 other intact occludin molecules between two neighboring SCs caused the recruitment of intact

359 and functional occludin to the same site to become impossible. Thus, the TJ permeability barrier

360 became disrupted.

361 There are three possible reasons for the decrease in protein expression. The first reason is 
362 protein degradation [32]. Occludin phosphorylation and ubiquitination regulate TJ [33]. The

363 western blot results showed that the molecular weight of occludin was consistent with the actual

364 size, suggesting that occludin did not degrade. This result indicates that 22AA did not cause

365 occludin ubiquitination. The second potential reason is cell apoptosis because cell apoptosis

366 prevents cells from expressing relevant proteins [34, 35]. Therefore, SC and spermatid apoptosis

367 might lead to a decrease in occludin expression. The third reason could be inhibition of

transcription or translation by noncoding RNA [6]. To investigate the occludin downregulation

mechanism, we analyzed the expression of several microRNAs (miRNAs) that can target

occludin (data not shown), among which only the expression of miR-122-5p was associated with

occludin expression. miR-122-5p is encoded on chromosome $18 \mathrm{q} 21.31$ and is derived from the

hcr gene transcript. miR-122-5p plays an important role in cell cycle regulation, cell proliferation

and cell apoptosis [36] and is associated with multiple diseases [36-39]. Previously, we analyzed

the correlation between miR-122-5p and occludin protein, and the results showed that miR-122-

$5 p$ was negatively correlated with occludin expression [40]. Our other recent results showed that

miR-122-5p regulates occludin expression through the AACACTCCA sequence of the occludin

3'UTR, thereby regulating the formation and tightness of TJ between SCs (submitted). We

further employed real-time RT-PCR to assess whether 22AA affects miR-122-5p expression.

The current results showed that the expression level of miR-122-5p gradually increased from 0 to

$27 \mathrm{D}$ and then began to decrease. The change in the miR-122-5p expression level was opposite

that of occludin. Correlation analysis showed a significant negative correlation between miR-

122-5p and occludin expression from 0 to 47D (Figure 3B ). These results indicate that 22AA 
383

384 385

increased the expression of miR-122-5p, which mediated downregulation of occludin expression, thereby causing disintegration of TJ. However, the mechanism by which 22AA regulates mir122-5p expression remains to be further studied.

To investigate the causes of $\mathrm{SC}$ and spermatid dysfunction, Bax was used as an apoptosis marker protein to analyze cell apoptosis in seminiferous tubules. Bax, belonging to the Bcl-2 gene family, is the most important apoptotic gene in humans. The encoded Bax protein forms heterodimers with Bcl-2 and has an inhibitory effect on Bcl-2. Bax is one of the most important apoptosis-promoting genes [41]. Bax expression is also closely related to spermatogenesis [42]. In the present study, Bax expression was found in the seminiferous tubules of the control group at a low expression level of $11.373 \pm 10.532$. Yan et al. also found that Bax was expressed in various types of cells in normal testicular tissues [43]. At 7D, the expression level of Bax was $16.783 \pm 10.157$, which was higher than that in the control group, indicating that the cells in seminiferous tubules began to undergo apoptosis. However, at this stage, the structure of SCs and spermatids in the tubules was relatively intact. The expression level of Bax increased to $20.521 \pm 5.781$ and $30.253 \pm 12.274$ at $17 \mathrm{D}$ and $27 \mathrm{D}$, respectively. At this stage, the tubules were filled with a large amount of red Bax, no blue nuclei were observed, and the intercellular boundary had completely disappeared, indicating that all the cells in the seminiferous tubules had already undergone apoptosis. At 37D, the expression level of Bax was slightly lower than at 27D. In the basal layer of the seminiferous tubules, blue nuclei began to appear, indicating that apoptosis had slowed and the spermatids and SCs of the seminiferous tubules had started to recover. At 47D, the expression level of Bax was not significantly different from that in the 
404

405

406

407

408

409

410

411

412

413

414

415

416

417

418

419

420

421

422

423

424

control group, and the structure and number of spermatids and SCs in seminiferous tubules were not different from those in the control group, indicating that spermatogenesis had fully recovered.

These results suggest that 22AA can induce apoptosis in seminiferous tubules before 27D.

To analyze the effect of 22AA on sperm count, the epididymis was extracted at $0,7,17,27$,

37, or 47 days. Sperm density was analyzed after the epididymis was shredded. The results are

shown in Figure 4A. The highest sperm density in the control group was $750.114 \times 10^{4} / \mathrm{mL}$, and

then, the density gradually decreased, dropping to $164.278 \times 10^{4} / \mathrm{mL}$ at $27 \mathrm{D}$; subsequently, the

sperm density gradually increased to $283.114 \times 10^{4} \mathrm{~mL}$ at 47D. However, compared with the

control group, the sperm density was greatly decreased. The decrease in sperm count may be due

to the following reasons. First, high expression of Bax promotes the apoptosis of type A spermatogonial stem cells [42], thereby reducing spermatogenesis. Second, after the BTB is destroyed, immune cells enter the seminiferous tubules and engulf many sperm [44]. Third, sperm undergo apoptosis or autophagy in the epididymis $[45,46]$. Further analysis is needed to determine which reason explains the decrease in sperm count. To analyze the effect of 22AA on litter size, male mice were co-caged with female mice at $0,7,17,27,37$, or 47 days. The number of embryos in the uterus on the 14th day of pregnancy was used to measure fertility. The highest litter size in the control group was 12 , but litter size decreased gradually to seven at 27D. Afterwards, the litter size gradually increased to 6.67 at 47D.

In summary, this study investigated the effect of $22 \mathrm{AA}$ on TJ by using a $22 \mathrm{AA}$-induced TJ destruction animal model. The results showed that before $27 \mathrm{D}, 22 \mathrm{AA}$ promoted $\mathrm{SC}$ and spermatid apoptosis, downregulated occludin, upregulated miR-122-5p, and decreased sperm 
425 density and litter size. After 27D, the occludin expression increased, miR-122-5p expression

426 decreased, both sperm density and litter size rebounded, cell apoptosis stopped, and

427 spermatogenesis began to recover. Therefore, it can be concluded that 22AA destroys TJ by

428 downregulating occludin and inducing cell apoptosis. After 27D, TJ and spermatogenesis

429 functions return to normal.

430

431

432

433

434

435

436

437

438

439

440

441

442

443

444

445

446

447

448

449

450

451

452

453

454

455

456

457

458

\section{Reference}

1. Chi X, Zhao X, Wang W, Niu Y, Cheng M, Liu X, Cui S, Yang W: Fusion expression of Occludin extracellular loops and an alpha-helical bundle: A new research model for tight junction. Plos One 2017, 12:e0175516.

2. Lui WY, Lee WM, Cheng CY: Transforming growth factor-beta3 perturbs the inter-Sertoli tight junction permeability barrier in vitro possibly mediated via its effects on occludin, zonula occludens-1, and claudin11. Endocrinology 2001, 142:1865-1877.

3. Li N, Mruk DD, Lee WM, Wong CKC, Cheng CY: Is toxicant-induced Sertoli cell injury in vitro a useful model to study molecular mechanisms in spermatogenesis? Semin Cell Dev Biol 2016, 59:141-156.

4. Tanaka M, Chiba K, Okada K, Fujisawa M: Effect of mirabegron on tight junction molecules in primary cultured rat Sertoli cells. Andrologia 2019, 51.

5. Ramos-Trevino J, Bassol-Mayagoitia S, Ruiz-Flores P, Espino-Silva PK, Saucedo-Cardenas O, Villa-Cedillo SA, Nava-Hernandez MP: In Vitro Evaluation of Damage by Heavy Metals in Tight and Gap Junctions of Sertoli Cells. DNA Cell Biol 2017, 36:829-836.

6. Feng H, Park JS, Zhai RG, Wilson ACC: microRNA-92a regulates the expression of aphid bacteriocytespecific secreted protein 1. BMC Res Notes 2019, 12:638.

7. Wiebe JP, Kowalik A, Gallardi RL, Egeler O, Clubb BH: Glycerol disrupts tight junction-associated actin microfilaments, occludin, and microtubules in Sertoli cells. J Androl 2000, 21:625-635.

8. Hew KW, Heath GL, Jiwa AH, Welsh MJ: Cadmium in vivo causes disruption of tight junction-associated microfilaments in rat Sertoli cells. Biol Reprod 1993, 49:840-849.

9. Chung NP, Mruk D, Mo MY, Lee WM, Cheng CY: A 22-amino acid synthetic peptide corresponding to the second extracellular loop of rat occludin perturbs the blood-testis barrier and disrupts spermatogenesis reversibly in vivo. Biol Reprod 2001, 65:1340-1351.

10. Liu C, Duan Z, Guan Y, Wu H, Hu K, Gao X, Yuan F, Jiang Z, Fan Y, He B, Wang, S, : Increased expression of tight junction protein occludin is associated with the protective effect of mosapride against aspirininduced gastric injury. Exp Ther Med 2018, 15:1626-1632.

11. Fanning AS, Jameson BJ, Jesaitis LA, Anderson JM: The tight junction protein ZO-1 establishes a link between the transmembrane protein occludin and the actin cytoskeleton. J Biol Chem 1998, 273:2974529753.

PeerJ reviewing PDF | (2020:07:51114:2:1:NEW 18 Sep 2020) 
459 12. Gonzalez-Mariscal L, Namorado MC, Martin D, Luna J, Alarcon L, Islas S, Valencia L, Muriel P, Ponce L, Reyes JL: Tight junction proteins ZO-1, ZO-2, and occludin along isolated renal tubules. Kidney Int 2000, 57:2386-2402.

13. Furuse M, Sasaki H, Fujimoto K, Tsukita S: A single gene product, claudin-1 or -2 , reconstitutes tight junction strands and recruits occludin in fibroblasts. J Cell Biol 1998, 143:391-401.

14. McCarthy KM, Skare IB, Stankewich MC, Furuse M, Tsukita S, Rogers RA, Lynch RD, Schneeberger EE: Occludin is a functional component of the tight junction. J Cell Sci 1996, 109 ( Pt 9):2287-2298.

15. Du D, Xu F, Yu L, Zhang C, Lu X, Yuan H, Huang Q, Zhang F, Bao H, Jia L, Wu X, Xueliang Zhu, Zhang X,

16. Ghassemifar MR, Sheth B, Papenbrock T, Leese HJ, Houghton FD, Fleming TP: Occludin TM4(-): an isoform

24. D'Aurora M, Ferlin A, Di Nicola M, Garolla A, De Toni L, Franchi S, Palka G, Foresta C, Stuppia L, Gatta V: of the tight junction protein present in primates lacking the fourth transmembrane domain. J Cell Sci 2002, 115:3171-3180.

17. Wong $\mathrm{CH}$, Mruk DD, Lee WM, Cheng CY: Targeted and reversible disruption of the blood-testis barrier by an FSH mutant-occludin peptide conjugate. FASEB J 2007, 21:438-448.

18. Bamforth SD, Kniesel U, Wolburg H, Engelhardt B, Risau W: A dominant mutant of occludin disrupts tight junction structure and function. J Cell Sci 1999, 112 ( Pt 12):1879-1888.

19. Medina R, Rahner C, Mitic LL, Anderson JM, Van Itallie CM: Occludin localization at the tight junction requires the second extracellular loop. J Membr Biol 2000, 178:235-247.

20. Gu JM, Lim SO, Park YM, Jung G: A novel splice variant of occludin deleted in exon 9 and its role in cell apoptosis and invasion. FEBS J 2008, 275:3145-3156.

21. Wong V, Gumbiner BM: A synthetic peptide corresponding to the extracellular domain of occludin perturbs the tight junction permeability barrier. J Cell Biol 1997, 136:399-409.

22. Langbein L, Pape UF, Grund C, Kuhn C, Praetzel S, Moll I, Moll R, Franke WW: Tight junction-related structures in the absence of a lumen: occludin, claudins and tight junction plaque proteins in densely packed cell formations of stratified epithelia and squamous cell carcinomas. Eur J Cell Biol 2003, 82:385400.

23. Malminen M, Koivukangas V, Peltonen J, Karvonen SL, Oikarinen A, Peltonen S: Immunohistological distribution of the tight junction components ZO-1 and occludin in regenerating human epidermis. $\mathrm{Br} \mathrm{J}$ Dermatol 2003, 149:255-260. Deregulation of sertoli and leydig cells function in patients with klinefelter syndrome as evidenced by testis transcriptome analysis. Bmc Genomics 2015, 16.

25. Tian R, Yang S, Zhu Z, Wang J, He Z, Li Z: Human Germ Cell Secreting Factor Nodal Regulates Sertoli Cell Functions. Fertil Steril 2014, 102:E355-E355.

26. Rebourcet D, Wu JX, Cruickshanks L, Smith SE, Milne L, Fernando A, Wallace RJ, Gray CD, Hadoke PWF, Mitchell RT, O'Shaughnessy PJ, Smith LB: Sertoli Cells Modulate Testicular Vascular Network Development, Structure, and Function to Influence Circulating Testosterone Concentrations in Adult Male Mice. Endocrinology 2016, 157:2479-2488.

27. Nagai T, Mizuno K, Hayashi Y, Kamisawa H, Moritoki Y, Nishio H, Kurokawa S, Nakane A, Maruyama T, Yasui T: Relationship between Sertoli Cell Function and Differentiation Process of Spermatogonial Stem 
Cells in Cryptorchid Testes. J Urology 2016, 195:E586-E586.

28. Nicholls PK, Stanton PG, Chen JL, Olcorn JS, Haverfield JT, Qian HW, Walton KL, Gregorevic P, Harrison CA: Activin Signaling Regulates Sertoli Cell Differentiation and Function. Endocrinology 2012, 153:6065-6077.

29. Wang X, Adegoke EO, Ma MJ, Huang FS, Zhang H, Adeniran SO, Zheng P, Zhang GX: Influence of Wilms' tumor suppressor gene WT1 on bovine Sertoli cells polarity and tight junctions via non-canonical WNT signaling pathway. Theriogenology 2019, 138:84-93.

30. Zalata AA, Mokhtar N, Atwa A, Khaled M, Shaker OG: The Role of Protamine 2 Gene Expression and Caspase 9 Activity in Male Infertility. J Urology 2016, 195:796-800.

31. Kleene KC, Bagarova J: Comparative genomics reveals gene-specific and shared regulatory sequences in the spermatid-expressed mammalian Odf1, Prm1, Prm2, Tnp1, and Tnp2 genes. Genomics 2008, 92:101106.

32. Majolee J, Kovacevic I, Hordijk PL: Ubiquitin-based modifications in endothelial cell cell contact and inflammation. Journal of Cell Science 2019, 132.

33. Murakami T, Felinski EA, Antonetti DA: Occludin phosphorylation and ubiquitination regulate tight junction trafficking and vascular endothelial growth factor-induced permeability. I Biol Chem 2009, 284:21036-21046.

34. Schiffmann LM, Gobel H, Loser H, Schorn F, Werthenbach JP, Fuchs HF, Plum PS, Bludau M, Zander T, Schroder W, Bruns CJ, Kashkar H, Quaas A, Gebauer F: Elevated X-linked inhibitor of apoptosis protein (XIAP) expression uncovers detrimental prognosis in subgroups of neoadjuvant treated and T-cell rich esophageal adenocarcinoma. BMC Cancer 2019, 19:531.

35. Liu C, Liang B, Wang Q, Wu J, Zou MH: Withdrawal: Activation of AMP-activated protein kinase alpha1 alleviates endothelial cell apoptosis by increasing the expression of anti-apoptotic proteins $\mathrm{Bcl}-2$ and Survivin. J Biol Chem 2020, 295:671.

36. Wang $Z$, Wang $X$ : miR-122-5p promotes aggression and epithelial-mesenchymal transition in triplenegative breast cancer by suppressing charged multivesicular body protein 3 through mitogen-activated protein kinase signaling. J Cell Physiol 2019.

37. Wen DY, Huang JC, Wang JY, Pan WY, Zeng JH, Pang YY, Yang H: Potential clinical value and putative biological function of miR-122-5p in hepatocellular carcinoma: A comprehensive study using microarray and RNA sequencing data. Oncol Lett 2018, 16:6918-6929.

38. Jiang $M$, Ma $W$, Gao $Y$, Jia K, Zhang $Y$, Liu H, Sun Q: IL-22-induced miR-122-5p promotes keratinocyte proliferation by targeting Sprouty2. Exp Dermatol 2017, 26:368-374.

39. Cortez-Dias N, Costa MC, Carrilho-Ferreira P, Silva D, Jorge C, Calisto C, Pessoa T, Robalo Martins S, de Sousa JC, da Silva PC, Manuela,Fiúza,António,Nunes, Diogo,Fausto,J,Pinto,Francisco,J,Enguita: Circulating miR-122-5p/miR-133b Ratio Is a Specific Early Prognostic Biomarker in Acute Myocardial Infarction. Circ J 2016, 80:2183-2191.

40. Zhu M, Fei L, Li D, Chen D: Correlation Analysis of miR-122-5p and Occludin with Sperm Density in Oligospermia Patients' Sperm. Clin Lab 2019, 65.

41. Szymona K, Dudzinska E, Karakula-Juchnowicz H, Gil-Kulik P, Chomik P, Swistowska M, Galaszkiewicz J, Kocki J: Analysis of the expression of BAX, BCL2, BIRC6, CASP3, CASP9 apoptosis genes during the first 
episode of schizophrenia. Psychiatr Pol 2019, 53:1293-1303.

42. Russell LD, Chiarini-Garcia H, Korsmeyer SJ, Knudson CM: Bax-dependent spermatogonia apoptosis is required for testicular development and spermatogenesis. Biol Reprod 2002, 66:950-958.

43. Yan W, Samson M, Jegou B, Toppari J: Bcl-w forms complexes with Bax and Bak, and elevated ratios of $\mathrm{Bax} / \mathrm{Bcl}-\mathrm{w}$ and $\mathrm{Bak} / \mathrm{Bcl}-\mathrm{w}$ correspond to spermatogonial and spermatocyte apoptosis in the testis. Mol Endocrinol 2000, 14:682-699.

44. Adegoke EO, Wang X, Wang H, Wang C, Zhang H, Zhang G: Selenium (Na2SeO3) Upregulates Expression of Immune Genes and Blood-Testis Barrier Constituent Proteins of Bovine Sertoli Cell In Vitro. Biol Trace Elem Res 2018, 185:332-343.

45. Grunewald S, Fitzl G, Springsguth C: Induction of ultra-morphological features of apoptosis in mature and immature sperm. Asian Journal of Andrology 2017, 19:533-537.

46. Sinkakarimi $\mathrm{MH}$, Solgi $\mathrm{E}$, Colagar $\mathrm{AH}$ : Subcellular partitioning of cadmium and lead in Eisenia fetida and their effects to sperm count, morphology and apoptosis. Ecotox Environ Safe 2020, 187. 


\section{Figure 1}

Figure 1. Effects of 22AA on the apoptosis of SCs, sperm cells and the localization and distribution of occludin .

Image $\mathrm{A}, \mathrm{G}$ and $\mathrm{M}$ were control panels, others image were different treatment panels.

Effects of 22AA on the apoptosis of SCs. Many SCs were present in the control group, with clear intercellular boundaries and a small amount of red Bax distribution $\square A \square$. The morphology and number of SCs at 7D were not significantly different from those of the control group(B). At 17D and 27D, there were no blue nuclei in the seminiferous tubules, and the intercellular boundary completely disappeared(C, D). The WT1 patch was scattered throughout the seminiferous tubules, with no cells present in the tubules. The tubules were filled with a large amount of red Bax. A few SCs began to appear in the basal layer of the seminiferous tubules at 37D (E). At 47D, the morphology, structure and number of SCs in the seminiferous tubules were not different from those in the control group(F).

Effect of 22AA on the apoptosis of sperm cells. Nearly $55 \pm 5$ spermatids were found in the seminiferous tubules of the control group $(G)$, and the expression level of Bax was $11.245 \pm 4.868$. At 7D, the spermatid count decreased to $15 \pm 3(\mathrm{H})$, and the expression of Bax increased to $19.569 \pm 6.158$. No spermatid was found in the seminiferous tubules at 17D (I) or 27D(J), and the expression levels of Bax increased to 23.467 \pm 5.327 and $31.353 \pm 13.139$, respectively. At 37D, a small amount of Prm2 was distributed in the seminiferous tubules(K), and the expression level of Bax decreased to $16.362 \pm 3.267$. At 47D, $20 \pm 4$ spermatids were rediscovered in the seminiferous tubules $(\mathrm{L})$, and the expression level of Bax was $10.176 \pm$ 1.682 , which was not different from the control level.

Effect of 22AA on the localization and distribution of occludin. Occludin was mainly located at TJ between the basement membrane of seminiferous tubules and SCs in the control group(M). At 7D, a small amount of occludin was distributed at TJ between the basement membrane of seminiferous tubule and SCs. However, the total number of cells in seminiferous tubules was less than that in the control group(N). At 17D and 27D, no cells with blue nuclei were found in the seminiferous tubules(O,P). From day 17 to day 27 , the expression of occludin gradually decreased. At 37D, occludin expression began to increase and was found to be distributed on the basement membrane of seminiferous tubules and $\mathrm{SCs}(\mathrm{Q})$. At 47D, occludin expression and distribution and the morphological structure of seminiferous tubules were highly similar to those in the control group(R). Barワ, $0.2 \mu \mathrm{m}$. 


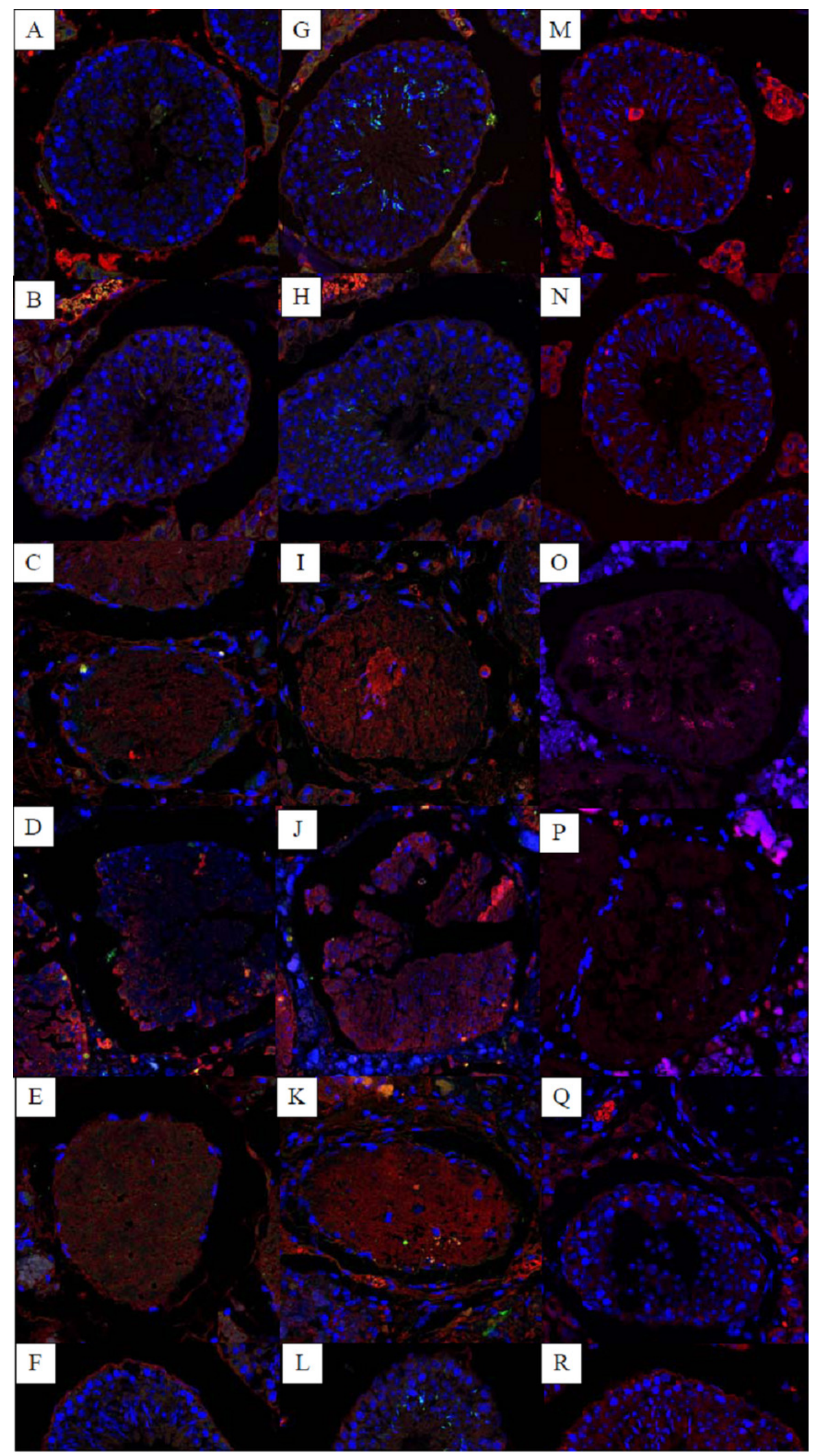




\section{Figure 2}

Effect of 22AA on the expression of occludin.

Representative western blot results are shown in Figure 2A. The relative occludin expression level was calculated using the occludin/ $\beta$-actin gray density ratio. The detailed values are shown in Figure 2B. In the control group, the expression value was 0.9967. From 7D to 27D, the occludin expression level in the 22AA group gradually decreased to 0.1621 , which was only $16.26 \%$ of that in the control group. Then, the expression level of occludin gradually increased to 0.3543 at $47 \mathrm{D}$, which was approximately one-third the normal expression level (35.54\%). Occludin expression was significantly different among the six groups $(P<0.05)$. The results showed that the expression level of occludin in the 22AA group at each time was significantly different from that in the control group. $\square P<0.01$. 

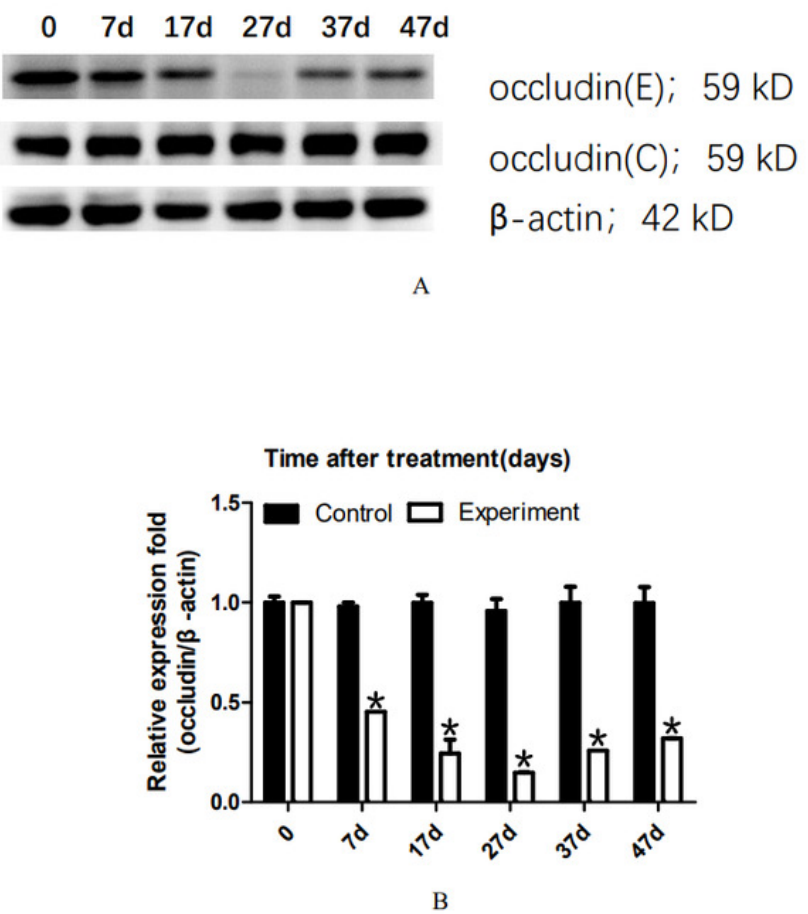


\section{Figure 3}

Effect of artificially synthesized 22AA on the expression of miR-122-5.

The expression levels of miR-122-5p in each group are shown in Figure 3A. The miR-122-5p expression level in the control group was 0.0408 and increased to 0.0539 at 7D. The miR-122-5p expression level in the 22AA group at 27D was the highest at 0.1293 . Then, miR-122-5p expression gradually decreased to 0.0867 at 47D but was still higher than that in the control group. The results showed that the miR-122-5p expression levels were significantly different among the six groups $(P<0.01)$. The correlation between miR-122-5p and occludin expression in each group was analyzed using Pearson correlation coefficient. The linear relationship is shown in Figure 3B. The results indicated that miR-122-5p and occludin expression are significantly negatively correlated $\left(R^{2}=-0.4905, P<0.01\right)$. $\square P<$ 0.01 . 


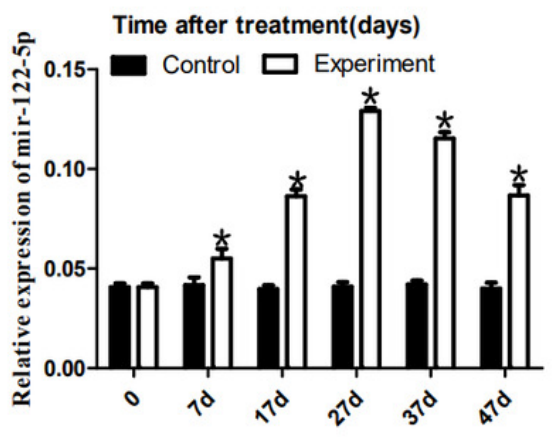

A

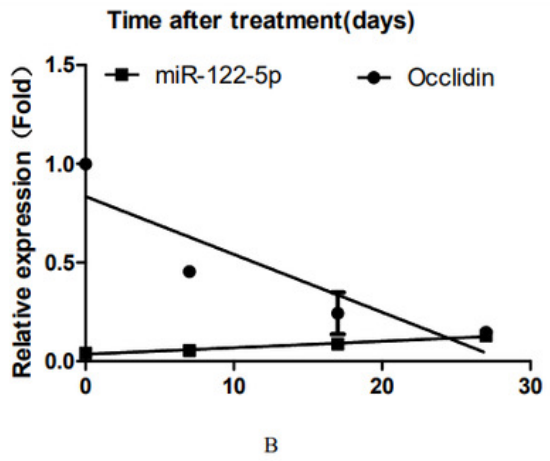




\section{Figure 4}

Effects of 22AA on sperm density and litter size.

The sperm density was analyzed after the epididymis was shredded. The results are shown in Figure 4A. The highest sperm density in the control group was $750.144 \times 10^{4} / \mathrm{mL}$, after which it gradually decreased. The sperm density decreased to $164.278 \times 10^{4} / \mathrm{mL}$ at $27 \mathrm{D}$ and then gradually increased to $283.114 \times 10^{4} \mathrm{~mL}$ at $47 \mathrm{D}$. The results showed a significant difference in sperm density among the six groups $(P<0.01)$. The number of embryos in the uterus on the 14th day of pregnancy was taken as a measure of fertility. The results are shown in Figure 4B. The largest litter size in the control group was 12, and then, litter size decreased gradually to 3.667 at $27 \mathrm{D}$. The litter size gradually increased to seven at 47D. The results showed that the difference in litter size among the six groups was significant. $\square \mathrm{P}<$ 0.01 . 

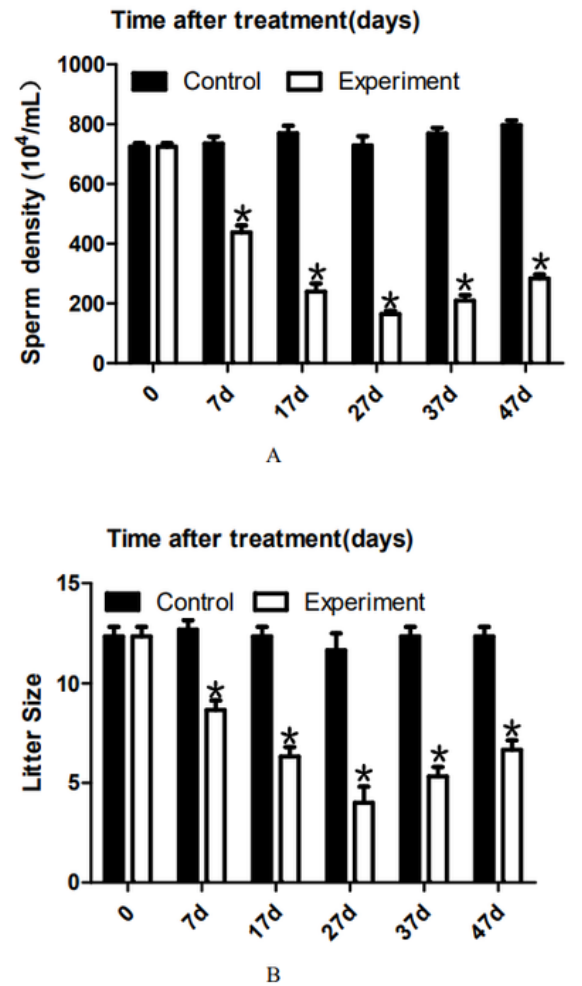


\section{Table $\mathbf{1}$ (on next page)}

Source of reagents in this study. 


\begin{tabular}{|c|c|}
\hline Types and sources of antibodies & Origin \\
\hline $\begin{array}{l}\text { Primary antibody: anti-Bax (rat } \\
\text { origin) }\end{array}$ & abcam, USA \\
\hline $\begin{array}{l}\text { Primary antibody: anti-WT1 (rabbit } \\
\text { origin) }\end{array}$ & abcam, USA \\
\hline $\begin{array}{l}\text { Primary antibody: anti-Prm2 (rabbit } \\
\text { origin) }\end{array}$ & Proteintech, USA \\
\hline $\begin{array}{l}\text { Secondary antibodies: anti-Alexa } \\
\text { Fluor CY3 (anti-mouse) }\end{array}$ & abcam, USA \\
\hline $\begin{array}{l}\text { Secondary antibody: anti-Alexa } \\
\text { Fluor } 488 \text { (anti-rabbit) }\end{array}$ & abcam, USA \\
\hline $\begin{array}{l}\text { Immunofluorescence primary } \\
\text { antibody (diluted) }\end{array}$ & Beyotime, China \\
\hline $\begin{array}{l}\text { Immunofluorescence secondary } \\
\text { antibody (diluted) }\end{array}$ & Beyotime, China \\
\hline PBS & ZSGB-BIO, Chia \\
\hline Paraformaldehyde & Shanghai Sangong \\
\hline Goat serum & HyClone, USA \\
\hline DAPI staining solution & Beyotime, China \\
\hline Anti-fluorescence quenching agent & Beyotime, China \\
\hline $\begin{array}{l}\text { Primary antibody: occludin (rabbit } \\
\text { origin) }\end{array}$ & abcam, USA \\
\hline $\begin{array}{l}\text { Secondary antibody: Anti-Alexa } \\
\text { Fluor cy3 (anti-rabbit) }\end{array}$ & abcam, USA \\
\hline Immunofluorescence & Beyotime, China \\
\hline
\end{tabular}




\begin{tabular}{|l|l|}
\hline antibody (diluted) & \\
\hline $\begin{array}{l}\text { Immunofluorescence secondary } \\
\text { antibody (diluted) }\end{array}$ & Beyotime, China \\
\hline PBS & ZSGB-BIO, Chia \\
\hline Paraformaldehyde & Shanghai Sangong \\
\hline Goat serum & HyClone, USA \\
\hline DAPI staining solution & Beyotime, China \\
\hline Anti-fluorescence quenching agent & Beyotime, China \\
\hline
\end{tabular}

2 